\title{
Plane wave propagation in generalized multiply connected acoustic filters
}

\author{
S. N. Panigrahi and M. L. Munjal ${ }^{\text {a) }}$ \\ Facility for Research in Technical Acoustics, Department of Mechanical Engineering, Indian Institute \\ of Science, Bangalore-560012, India
}

(Received 26 October 2004; revised 10 August 2005; accepted 12 August 2005)

\begin{abstract}
A generalized algorithm is developed for studying the sound wave propagation in a system of interconnected rigid walled acoustic filter elements. The algorithm is based on the transfer matrix approach of analysis. Interconnection between various elements is represented by a connectivity matrix. Equations of mass velocity continuity and pressure equilibrium at the interconnections are generated using this connectivity matrix and are solved to get the overall transfer matrix of the system. The algorithm used for generalized labeling of the network and computation of transmission loss is discussed. The algorithm is applied to investigate multiply connected automobile mufflers as a network of acoustic elements. Results for some configurations have been compared with those from the finite element model analysis and experiments cited in the literature. A parametric study with respect to some geometric variables is carried out. While the results are illustrated here for a few configurations, the approach holds for all kinds of combinations of acoustic elements with any degree of complexity. The acoustical similarity between apparently different networks is discussed. The approach is flexible to incorporate any other acoustic elements, provided the acoustic variables at the junctions of the element can be related by a transfer matrix a priori. (C) 2005 Acoustical Society of America. [DOI: 10.1121/1.2049127]
\end{abstract}

PACS number(s): 43.20.Mv [SFW]

Pages: 2860-2868

\section{INTRODUCTION}

Acoustic wave propagation through a combination of acoustic filter or muffler elements has been studied for a very long time in the history of acoustic filters. Study of two tubes in parallel connection, for varying lengths and crosssectional areas, has been carried out since the 19th century, first by Herschel and Quincke ${ }^{1,2}$ and later refined by Stewart and Lindsay ${ }^{3-5}$ in the early 20th century. Stewart's analysis, however, was a restricted one in a way that it forced the cross-sectional areas of the parallel ducts to be equal and the sum of the cross-sectional areas of the two branch ducts to be the same as that of the entrant and the exit duct. A general analytical expression for the transmission loss of a simple two-node, two-duct configuration [i.e., the Herschel-Quincke (HQ) tube] was developed and the numerical and experimental verification of the same was carried out by Selamet et al. ${ }^{6}$ In their work, the conservation equations for mass, momentum, and internal energy, coupled with the ideal gas equation of state were formulated and solved using numerical methods.

Historically, the use of the HQ tube has been restricted to the suppression of tones of specific frequencies. The sound attenuation that can be achieved by use of such tube networks has not been exploited as it has been argued that the attenuation bands are too narrow. ${ }^{7}$ But their attenuation bandwidth may be enhanced by removing the restrictions imposed by Stewart and by increasing the number of parallel ducts. Selamet and Easwaran ${ }^{8}$ developed a close form expression for the transmission loss characteristics and reso-

${ }^{a)}$ Electronic mail: munjal@mecheng.iisc.ernet.in nance locations for an $n$-duct configuration. The analysis, however, was restricted to the configuration where inlets and outlets of all the " $n$ " ducts were connected together, respectively.

The physical connection of two or more acoustic elements makes the pressure equal in all of the elements associated at the point of connection. In the present work, it is assumed that the lateral dimensions of all the elements are such that the disturbances (the higher order modes) that are created at the junction of elements or at any sharp bend that may be present, die out within a very small distance (as compared to the lengths of the converging tubes) from the junction or the bend for the whole frequency range of interest. So, the pressure field outside this small region of disturbance can be considered as plane in nature and this pressure has been considered equal in all the elements at the junctions. While using complex acoustic elements, the only care that must be taken is to ensure that plane wave assumption holds good in the frequency range of interest in all of the constituting elements of the network. Insofar as the present work is concerned, the walls of all the acoustic elements are assumed to be strictly rigid so as to avoid any dispersive effect in the waves carried through them. The summation of volume velocity is also zero at these points. Such points of connection of elements, hereafter, in analogy to the electrical circuitry terminology, are referred to as nodes in the present paper. Similarly, the acoustic element connecting any two nodes is referred to as a branch to keep the discussion general with respect to the elements constituting an acoustic filter (explicit mention of some of the used elements has been made in the next section). Such systems or acoustic filters with many nodes and branches are referred to as acoustic 
networks in the present paper. So, as an example, the configuration taken up by Selamet and Easwaran in their work $^{8}$ shall be referred to here as a 2-node, $n$-branch network of rigid walled ducts.

In the existing literature, generally, two methods, namely, the transfer matrix method and stiffness matrix method, are followed to analyze suchlike mufflers or any acoustic network in general. Chainlike networks similar to a muffler or a long duct with many changes of cross sectional area and many segments for which individual transfer matrices are known, have been dealt with as a cascade by means of the transfer matrix approach. ${ }^{9,10}$ Apart from the attempt to get a broad band sound attenuation, as in the case of automobile mufflers and multiply tuned quincke tubes, there is another objective of the analysis of networks of acoustic wave guides. In the gas piping systems, the knowledge of resonance frequencies is more important than the overall sound attenuation. Simplified analyses have been used to study resonance in pipelines. ${ }^{11} \mathrm{To}^{12,13}$ analyzed the acoustic propagation in piping systems by developing a computer program based on the transfer matrix approach. In applying his approach, one needs to first locate the subsystems with certain input/output combination. For example, one-inputone-output, one-input-two-output, two-input-one-output, etc. This task becomes very complicated when one deals with a fairly complex network of filter elements. Again, at times, it can be very difficult to distinguish a two-input-oneoutput subsystem from a one-input-two-output subsystem, and so on.

Craggs et al. ${ }^{14}$ have used the stiffness matrix method to analyze the attenuation behavior of sound waves in pipe networks. They have used a two-dimensional finite element model (FEM) of the bends and junctions of the network and combined these solutions with exact solutions for the pipe to study the overall sound attenuation in a certain network of pipes, which they have verified experimentally.

Recently, Dowling and Peat ${ }^{15}$ have used the transfer matrix approach to analyze silencers of general geometry. They have devised a path fraction algorithm to break the complete network into subsystems of simpler elements. This method uses a fork (similar to the one-input-two-output subsystem of $\mathrm{To}^{12}$ ) to connect a single input to two outputs. But, if a number of branches emerge from a single point and all of them are connected to some different locations, which in turn may be the confluence point of many other branches of the network, it may not be possible to break the network using only forks, particularly in the case of three-dimensional networks, which is common in all realistic gas piping systems.

The present paper suggests a transfer matrix based approach to analyze a generalized $m$-node, $n$-branch, two-port network of ducts (e.g., see Fig. 1). The fifth configuration of the figure below shows two ducts emerging from the same point and they seem to be overlapping. This confusion can be avoided if the network is visualized in a three-dimensional space. An example of such a configuration has been shown in Fig. 2 where several branches emerge from the same point of the network. So, the concept of line diagram has been used to represent the networks and is discussed in the next section. A scheme has been proposed to label the network. A connec-

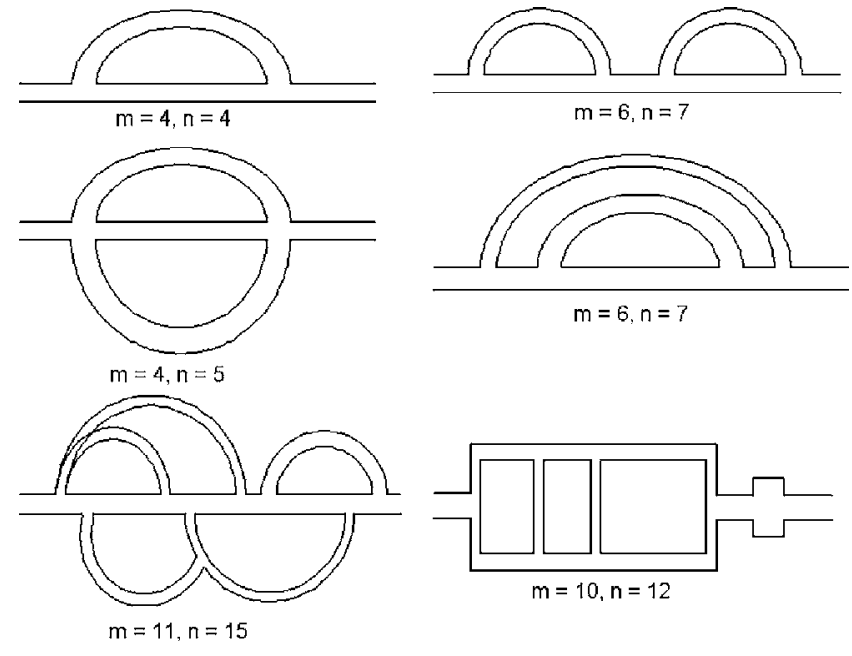

FIG. 1. Schematic of some representative $m$-node, $n$-branch acoustic filters.

tivity matrix is formed using the network labeling. The connectivity matrix gives an indication of acoustically similar networks with different physical appearance. An algorithm has been developed to use the connectivity matrix to automate the generation of the pressure balance equations, and volume-velocity continuity equations along with the proper transfer matrices between the nodes. The final transfer matrix, which relates the acoustic variables at the upstream and downstream points, is then evaluated from which the transmission loss can be calculated. ${ }^{9,10}$ Investigation of complex, multiply connected mufflers is carried out using the present method. This generalized network can be used to analyze many other acoustic elements which cannot be thought of as networks on a first look. Some such cases are analyzed to show the usefulness of this generalized network analysis.

The present study consists of four sections. Following this Introduction, the algorithm developed is discussed.
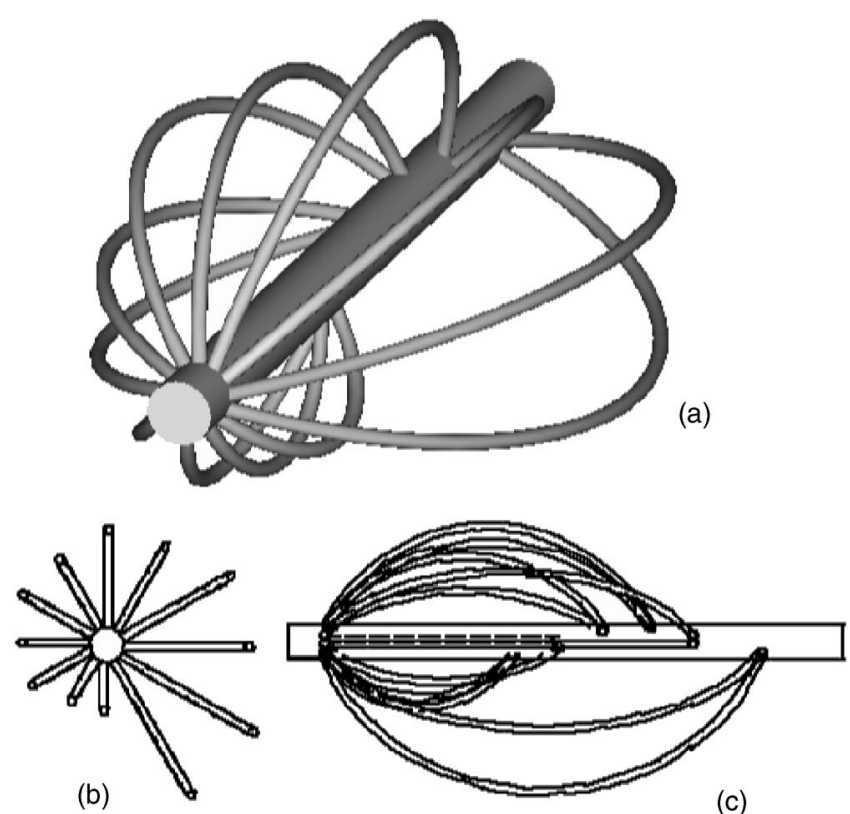

(c)

FIG. 2. (a) 3D view, (b) left side view, and (c) front view of a multiply tuned Quincke tube. 
Then, the predictions are validated and some configurations are analyzed to show the flexibility and usefulness of the approach. One section exclusively deals with the investigation of multiply connected mufflers. The study is then summarized and concluded with some final remarks.

\section{ALGORITHM}

As pointed out previously, some times it becomes difficult to comprehend from the two-dimensional representation of networks. The three-dimensional figures are not suitable for analysis either. So, the line diagram representation is used in the present work to represent the networks. A general $m$-node, $n$-branch network can be drawn by first noting the nodes of the network. As indicated above, a node represents a branching of a certain duct into several elements or the confluence of several elements at a specific point. Then the nodes can be connected by appropriate number of elements referred to as branches or edges as indicated in a preceding section. The lateral dimensions of these elements have been considered small so as to have the plane wave region up to the maximum frequency of interest. The medium inside the elements is assumed to be a gaseous fluid so that the rigidity assumption for the walls can be relied upon to a reasonable degree of accuracy. These elements can be simple rigid walled ducts, expansion chambers with or without any extended inlet/outlet, concentric tube resonators, duct with any number of mutually interacting perforated ducts, ${ }^{16}$ or for that matter, any other newly developed element. The theory of deriving the transfer matrices for many of these filter elements has been discussed in the literature. ${ }^{9,10}$ The detailed derivation of transfer matrix for some particular acoustic element may become very involved at times. The simplicity of the present method lies in the fact that the transfer matrix (derived rigorously) of such complex elements can also be used directly without going into the intricacies of the derivation. The cutoff frequency for the constituting elements, however, can be calculated from their geometrical parameters by referring to the respective literature.

A statement is worth noting regarding the abovementioned elements. Complex elements like concentric tube resonators or similar can be used directly in the network if the transfer matrix for the same is known a priori. Otherwise these elements can themselves be analyzed as networks made of simpler elements. The end terminations can be directly incorporated as separate elements and the transfer matrices for such terminations take into account the impedance of the termination. So, no special treatment is needed to handle such situations. However, the rigid terminations can be dealt with in a much simpler way. A rigid duct of very small crosssectional area (nearing zero) and a very large length (arbitrarily large) can be imagined to be emerging from such a termination and can be connected to any point of the network. When two or more such terminations exist, they can be connected to each other in this manner. This kind of treatment of the termination has been illustrated in subsequent sections. The lengths of the network branches need not be considered while drawing these line diagrams. An example of a line diagram is shown in Fig. 3 (the inlet and the outlet

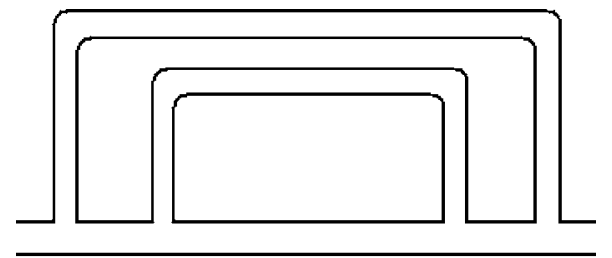

(a) Schematic diagram of a 4-node, 5-duct network

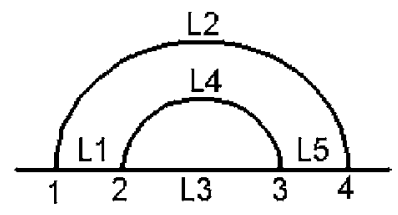

(b) Line diagram representation of the above network

FIG. 3. An example of line diagram representation of general network.

tubes have not been considered) with its corresponding twodimensional network representation. A sudden change in area can also be handled by introducing nodes at such discontinuities. Such configurations are analyzed later in a subsequent section.

Once the line diagram is prepared, the next step is to number the nodes. The nodes are numbered sequentially, starting from 1, and this is assigned to the inlet of the network. The numbering of intermediate nodes can be done arbitrarily except that the outlet of the network must be assigned the highest node number. The complete algorithm has been implemented through a computer program using MATLAB Version 6. In the data input stage, which is the first stage of the algorithm, the program involves gathering data regarding the connectivities among the different elements as user input with the help of the line diagram. It also involves gathering information about the type of elements for each connection, physical data (radii, lengths, perforation parameters, etc.) for each element, and then storing the complete information as an editable data file. When multiple interacting ducts are to be included in the network some more data (information about the nodes that are associated with the multiply interacting duct) must be entered so that the program can identify all the nodes which are associated with these interacting ducts. To enter this information correctly, the line diagram itself is modified with dotted lines (instead of solid lines) between the nodes which are part of these interacting nodes. Use of this kind of line diagram has been illustrated in subsequent sections through examples. This data file can later be edited manually to make small changes for some parametric study which would help in reducing the total designing time of any particular filter or muffler configuration. Each node may or may not be connected to all the other nodes, and between any pair of nodes there can be several branches. In the analysis stage, the program reads data from the stored file and generates the program variables. The connectivity information is stored in the form of a matrix, hereafter called the connectivity matrix. For the network shown in Fig. 3, this would look like the matrix $[\mathbf{C}]$ in Eq. $(1)$

S. N. Panigrahi and M. L. Munjal: Multiply connected acoustic filters 


$$
[\mathbf{C}]=\left[\begin{array}{cccc}
\times & 1 & 0 & 1 \\
1 & \times & 2 & 0 \\
0 & 2 & \times & 1 \\
1 & 0 & 1 & \times
\end{array}\right]
$$

The cross marks in the diagonal position suggests that each node is connected to itself. Nonzero values in other positions denote the number of branches between the pair of nodes, the pair being decided by the position in the matrix. For example, $[\mathbf{C}]_{23}=1$ indicates that there is only one branch connecting the pair of nodes 2 and 3, and so on. A zero at any position implies that the corresponding nodes are not connected directly. The upper diagonal part of this connectivity matrix is then traversed for getting the information about the number of branches between each pair of nodes, and labels are assigned to them sequentially as $L_{1}, L_{2}, \ldots$, etc., up to the last branch. For the present configuration, $[\mathbf{C}]_{12}=1,[\mathbf{C}]_{14}=1$ suggest that the name of the branches between nodes $1-2$ and $1-4$ is $L_{1}$ and $L_{2}$, respectively. Between nodes 2 and 3 there are two branches $\left([\mathbf{C}]_{23}=2\right)$ which are labeled as $L_{3}$ and $L_{4}$. This labeling can be done without any preference to any of the branches between these nodes. This labeling is done automatically through the program. The physical parameters are input at this stage of operation. The upstream and the downstream ends are also assigned to the corresponding branches. Once all this information is gathered, the transfer matrix equations are generated, assuming plane wave propagation, for each branch between their corresponding nodes. The state variables at the upstream and the downstream ends of each branch are related by the transfer matrix as

$$
\left\{\begin{array}{l}
p \\
v
\end{array}\right\}_{L_{i}}^{u}=[\mathbf{T M}]\left\{\begin{array}{l}
p \\
v
\end{array}\right\}_{L_{i}}^{d},
$$

where $p$ is the acoustic pressure and $v$ is the acoustic mass velocity. $[\mathbf{T M}]$ is the transfer matrix, superscripts " $u$ " and " $d$ " denote the upstream/downstream points and " $L_{i}$ " denotes the branch for which the equation is being written. The above equations could also have been written in terms of the progressive forward and rearward moving pressure waves. ${ }^{6}$ The advantage of writing in the transfer matrix form is that, any acoustic element (or a cascade of elements) for which transfer matrix is known can directly be incorporated between any pair of nodes; a branch does not have to be a uniform tube, duct or pipe. This makes the algorithm more general in terms of analyzing various networks with different acoustic elements. The equations are generated using the symbolic arithmetic so that final results can be evaluated in two different forms. One can assign numerical values to all parameters and solve the final equation numerically. Alternatively, the equations can be solved symbolically and later on the numerical values can be assigned. The second method generally takes much longer time to solve and is computationally more demanding on the resources. But once this solution is obtained the parametric studies become much easier and faster. So, if the network is made of tubes only, which happens to be the case in the current example, Eq. (2) becomes

$$
\left\{\begin{array}{l}
p \\
v
\end{array}\right\}_{L_{i}}^{u}=\left[\begin{array}{cc}
\cos \left(k L_{i}\right) & j Y_{i} \sin \left(k L_{i}\right) \\
\frac{j}{Y_{i}} \sin \left(k L_{i}\right) & \cos \left(k L_{i}\right)
\end{array}\right]\left\{\begin{array}{l}
p \\
v
\end{array}\right\}_{L_{i}}^{d},
$$

where $k=\omega / a_{0}$ and $Y_{i}=a_{0} / S_{i} . k$ is the wave number and $Y_{i}$ denotes the characteristic impedance of $i$ th branches labeled as $L_{i}$. $\omega$ is the driving frequency, $a_{0}$ is the speed of sound in air, and $S_{i}$ represents the cross-sectional area of $i$ th branch. The transfer matrix equations for the first duct of the present example that are generated in the program are given as

$$
\begin{aligned}
& p_{u}^{L_{1}}-\cos \left(k L_{1}\right) p_{d}^{L_{1}}-\frac{j a_{0} \sin \left(k L_{1}\right) v_{d}^{L_{1}}}{S_{1}}=0, \\
& v_{u}^{L_{1}}-\frac{j S_{1} \sin \left(k L_{1}\right) p_{d}^{L_{1}}}{a_{0}}-\cos \left(k L_{1}\right) v_{d}^{L_{1}}=0 .
\end{aligned}
$$

Each such equation introduces four new unknowns in the total list of unknowns.

Conditions for pressure equilibrium at each node or junction of acoustic elements can be expressed as Eq. (6) and the corresponding equations for the case at hand are given as Eq. (7),

$p_{u, d}^{L_{i}}=p_{u, d}^{L_{j}}$,

$p_{d}^{L_{1}}-p_{u}^{L_{3}}=0, \quad p_{d}^{L_{1}}-p_{u}^{L_{4}}=0, \quad p_{d}^{L_{3}}-p_{d}^{L_{4}}=0, \quad p_{d}^{L_{3}}-p_{u}^{L_{5}}=0$.

Here, $j$ traverses through all the elements (except the element with the lowest serial number) present at a particular junction of elements and $i$ represents the element which bears the lowest serial number among the set of elements present at that junction. The "up" or the "down" status is read from the matrix generated during the file reading process to obtain the connectivity matrix. Equation (6) represents the pressure equilibrium equation at the intermediate nodes. The corresponding equations for the inlet and the outlet ports, respectively, are as follows:

$$
\begin{aligned}
& p_{u}=p_{u}^{L_{i}}, \\
& p_{d}=p_{d}^{L_{i}} .
\end{aligned}
$$

For the present example network, the pressure equilibrium equations are given as

$$
\begin{aligned}
& p_{u}-p_{u}^{L_{1}}=0, \quad p_{u}-p_{u}^{L_{2}}=0, \\
& p_{d}-p_{d}^{L_{5}}=0, \quad p_{d}-p_{d}^{L_{2}}=0 .
\end{aligned}
$$

Continuity of mass velocity (assuming uniform density) at the nodes is expressed as

$$
v_{u}+v_{u}^{L_{b 1}}+\cdots+v_{u}^{L_{b n}}=0
$$

at the inlet of the network, as 


$$
v_{d}+v_{d}^{L_{b 1}}+\cdots+v_{d}^{L_{b n}}=0
$$

at the outlet of the network, and as

$$
v_{u, d}^{L_{b_{1}}} \pm v_{u, d}^{L_{b 2}} \cdots \pm v_{u, d}^{L_{b n}}=0
$$

at the intermediate junctions. For the present example network (Fig. 3), the equations for continuity of volume velocity are given as

$$
v_{u}+v_{u}^{L_{1}}+v_{u}^{L_{2}}=0, \quad v_{d}+v_{d}^{L_{2}}+v_{d}^{L_{5}}=0,
$$

for the inlet and outlet on the network, respectively, and

$$
v_{d}^{L_{1}}-v_{u}^{L_{3}}-v_{u}^{L_{4}}=0, \quad v_{d}^{L_{3}}+v_{d}^{L_{4}}-v_{u}^{L_{5}}=0
$$

for the intermediate nodes.

It is worth noting that addition of each new branch introduces four more equations. For example, when a new branch is added to a single tube having six equations, to form the Herschel-Quincke tube, the number of equations increases to 10 . So, for an $n$-duct configuration the total number of equations would be $4 n+2$. Each transfer matrix equation introduces four additional unknown variables. Therefore, there would be $4 n$ unknowns. The state variables at the inlet and the outlet ports, namely $p_{u}, v_{u}, p_{d}$, and $v_{d}$, add four more unknowns making the total number of unknowns $4 n+4$. On eliminating the intermediate unknown variables, the pressure and the volume velocity at the inlet of the network can be expressed in terms of those at the outlet of the network. Then, setting $v_{d}=0$ and $p_{d}=1$ in the pressure equation, $T_{11}$ can be found out. Similarly, the other three terms, namely, $T_{12}, T_{21}$, and $T_{22}$ of the transfer matrix can also be evaluated. Then the two upstream variables at the inlet port can be expressed in terms of the two downstream variables at the outlet port as

$$
\left\{\begin{array}{l}
p \\
v
\end{array}\right\}_{u}=\left[\begin{array}{ll}
T_{11} & T_{12} \\
T_{21} & T_{22}
\end{array}\right]\left\{\begin{array}{l}
p \\
v
\end{array}\right\}_{d},
$$

where $T_{i j}(i, j=1,2)$ are known as the four-pole parameters of the system. The transmission loss for the network can be expressed in terms of these four-pole parameters as ${ }^{9}$

$$
\mathrm{TL}_{a}=20 \log _{10} \frac{\left(T_{11}+T_{12} / Y+Y T_{21}+T_{22}\right)}{2},
$$

where $Y$ is the characteristic impedance of the upstream duct (inlet port) or the downstream duct (outlet port), assumed to be equal in Eq. (18).

\section{VALIDATION OF THE ALGORITHM}

Some self-consistency checks have been carried out for validation of the algorithm described above. For a 2-node, 2-branch $\left(L_{1}, L_{2}\right.$ being the lengths of the two branches) network the transmission loss has been calculated. This network is supposed to be acoustically identical to a simple expansion chamber $^{8}$ when the two branches become equal in length, the area of expansion chamber being equal to the combined area of the two ducts. In this case the expression for TL is given by Davis et al. ${ }^{7}$ as

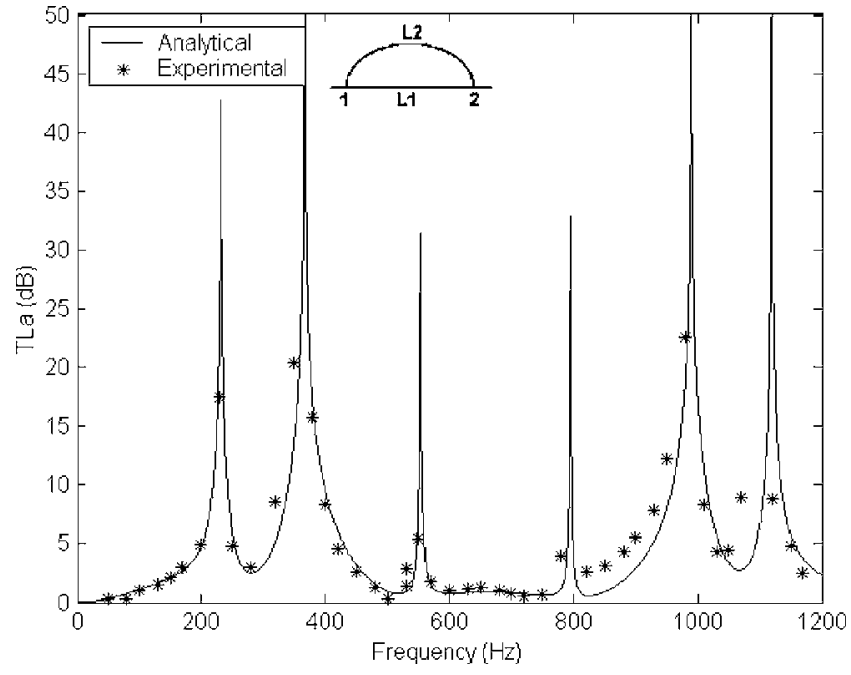

FIG. 4. Transmission loss spectra for the network $\left(L_{1}=0.3935 \mathrm{~m}, L_{2}\right.$ $\left.=0.9065 \mathrm{~m}, r_{1}=0.0243 \mathrm{~m}, r_{2}=0.01905 \mathrm{~m}\right)$ investigated by Selamet et al. (Ref. 6). Comparison of network analysis and the experimental results.

$$
\mathrm{TL}=10 \log _{10}\left[1+\frac{1}{4}(m-1 / m)^{2} \sin ^{2}(k l)\right],
$$

where $m$, the area ratio, is given by $\left(S_{1}+S_{2}\right) / S_{u} . S_{1}, S_{2}$ are the area of cross section of the two branches and $S_{u}$ is the crosssectional area of the upstream duct. The TL predicted from the present network analysis has been verified to be identical to that from Eq. (19) as expected. The TL curve for unequal lengths $\left(L_{2} \neq L_{1}\right)$ has been observed to be above the TL curve for equal lengths of the branches. This comparison brings out the importance of the role of unequal branch lengths in wave cancellation.

Figure 4 shows the comparison of the predicted results with the experimental ${ }^{6}$ ones for a 2-node, 2-branch network $\left(L_{1}=0.3935 \mathrm{~m}, \quad L_{2}=0.9065 \mathrm{~m}, \quad r_{1}=0.0243 \mathrm{~m}, \quad r_{2}\right.$ $=0.01905 \mathrm{~m}$ ) with unequal lengths and unequal crosssection areas of the branch ducts.

The present approach has been used to analyze a 2-node, $n$-branch network as a special case of the $m$-node, $n$-branch network. The results obtained for 2-node, 2- and 3-branch configurations $\left(L_{1}=0.4 \mathrm{~m}, \quad L_{3}=0.8 \mathrm{~m}, \quad r_{1}=0.025 \mathrm{~m}, \quad r_{2}\right.$ $=0.025 \mathrm{~m}, r_{3}=0.025 \mathrm{~m}$ ) have been checked to have an exact match with those of Selamet et al. ${ }^{8}$ as shown in Fig. 5. After validating for a 2 -node, $n$-branch configuration, the approach has been used to predict transmission loss for an $m$-node, $n$-branch (6-node, 7-branch) network of tubes. The experimental results obtained by Craggs et al. ${ }^{14}$ for the network have been compared in Fig. 6 with those obtained from the present approach. The two can be seen to be matching well. The dimensions (in meters) and other parameters used here are the same as those used in Ref. 14 and are as follows:

$$
L_{1}=0.2, \quad L_{2}=L_{3}=L_{4}=L_{5}=L_{6}=0.453 .
$$

The branch denoted by $L_{7}$, in fact, is an expansion chamber with inlet length of $0.1555 \mathrm{~m}$, expanded chamber length of $0.05 \mathrm{~m}$ and area expansion ratio of 3.25 to confirm with the dimensions used in the cited reference. They have used a square cross section for the tubes of the network. For the present study, tubes with the same area of cross 


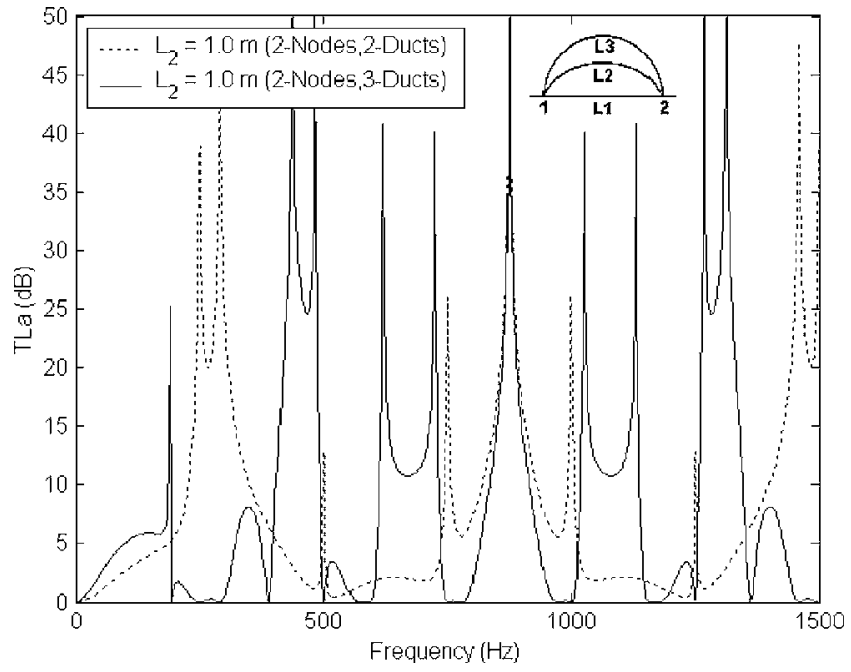

FIG. 5. Transmission loss spectra for the network $\left(L_{1}=0.4 \mathrm{~m}, L_{3}=0.8 \mathrm{~m}\right.$, $r_{1}=0.025 \mathrm{~m}, r_{2}=0.025, r_{3}=0.025 \mathrm{~m}$ ) investigated by Selamet et al. (Ref. 8) predicted from the present analysis.

section (with $r=0.027 \mathrm{~m}$ ) have been considered in modeling the equivalent general network.

\section{SOME PARAMETRIC STUDIES}

A general 4-node, 5-branch network is shown in Fig. 7(a). Figures 7(b) and 7(c) show similar networks with expansion chambers in one and two of their branches, respectively. All these networks can be analyzed as 4-node networks if the transfer matrix for the branches with expansion chambers is used directly between the corresponding nodes. They can also be analyzed by introducing more number of nodes in the branches and this method has been used here for the analysis. The line diagrams for the above configurations are shown in the inset of Fig. 8. The curves show the transmission loss predicted for the networks shown in the inset

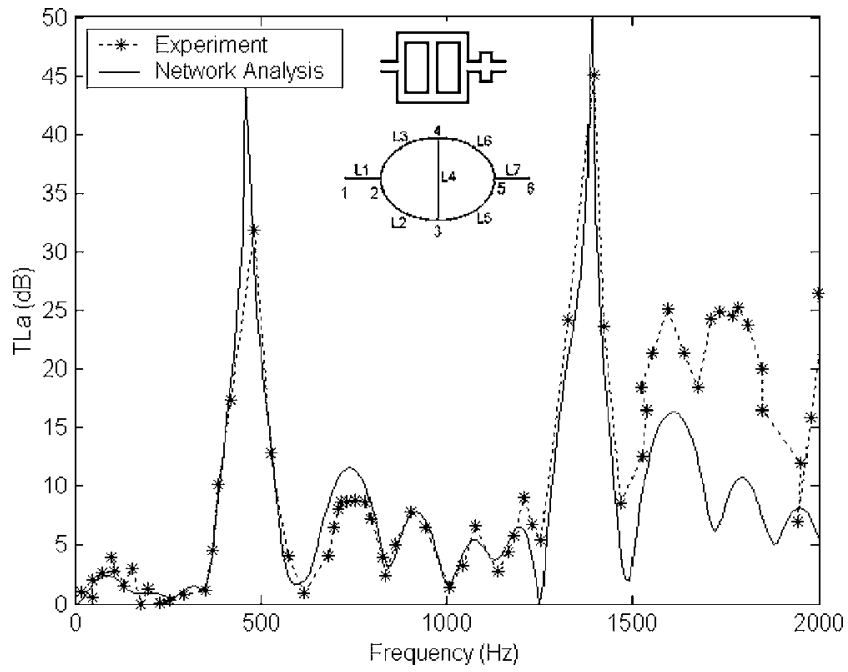

FIG. 6. Transmission loss spectrum for a 6-node, 7-branch, acoustic filter $\left(L_{1}=0.2 \mathrm{~m}, \quad L_{2}=L_{3}=L_{4}=L_{5}=L_{6}=0.453 \mathrm{~m}, \quad r=0.027 \mathrm{~m}, \quad L_{7}=\right.$ expansion chamber with inlet length of $0.1555 \mathrm{~m}$, expansion ratio $=3.25$, length of expanded chamber $=0.05 \mathrm{~m}$ ). Comparison of predicted results from present analysis with the experimental results of Craggs et al. (Ref. 14).

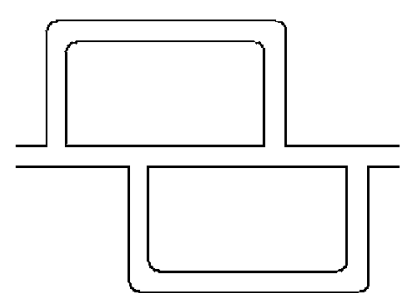

(a) Simple Network with no expansion chamber

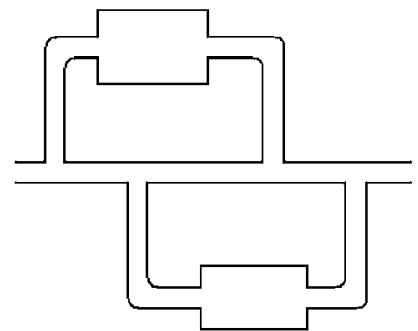

(c) Network with two expansion chambers

FIG. 7. A network with (a) zero, (b) one, and (c) two expansion chambers on the branches.

comprising a number of tubes and expansion chambers. For the configuration (a) of Fig. 8, the dimensions (in meters) used are

$r_{u}=r_{1}=r_{2}=r_{3}=r_{4}=r_{5}=r_{d}, \quad r_{u}=0.02$,

$L_{1}=0.1, \quad L_{2}=2 L_{1}, \quad L_{3}=1.5 L_{1}, \quad L_{4}=2.5 L_{1}, \quad L_{5}=L_{1}$.

The configuration (b) shown in the inset of Fig. 8 consists of an expansion chamber on its upper branch between nodes 3 and 4 (denoted by $L_{5}$ ). Dimensions for this configuration are considered in such a way that the total length of the upper branch of configurations (a) and (b) remains the same, i.e.,

$$
\left(L_{2}\right)_{a}=\left(L_{2}+L_{5}+L_{6}\right)_{b}
$$

where $L_{2}=L_{6}=L_{1} / 2, L_{5}=L_{1}, \quad$ and $r_{5}=3 r_{u}$.

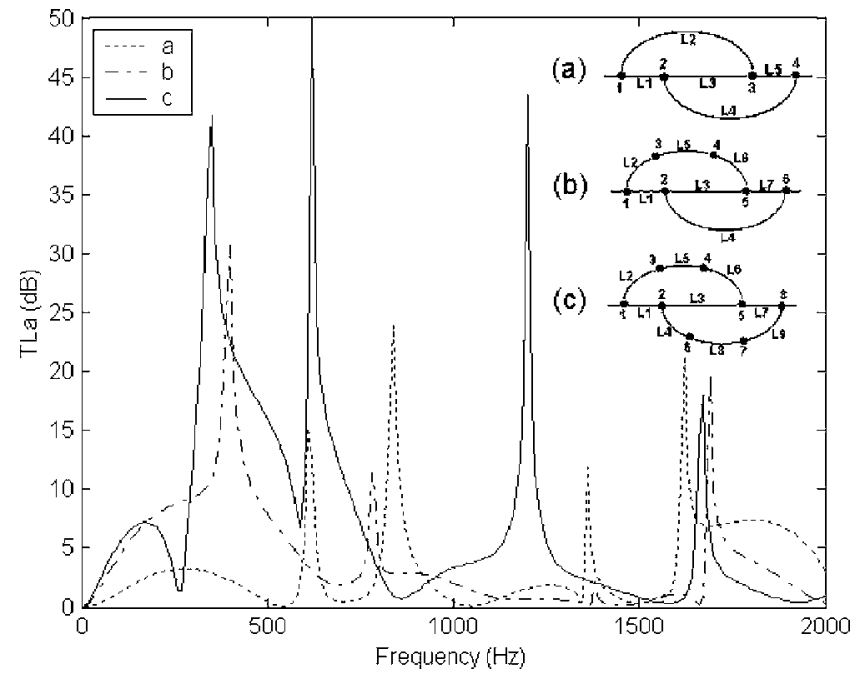

FIG. 8. Comparison of the axial transmission loss spectra of networks containing (a) zero, (b) one, and (c) two expansion chambers on the branch ducts. (a) $r_{u}=r_{1}=r_{2}=r_{3}=r_{4}=r_{5}=r_{d}, r_{u}=0.02 \mathrm{~m}, L_{1}=0.1 \mathrm{~m}, L_{2}=2 L_{1}, L_{3}$ $=1.5 L_{1}, L_{4}=2.5 L_{1}, L_{5}=L_{1}$; (b) $L_{2}$ of (a) $=\left(L_{2}+L_{5}+L_{6}\right)$ of (b); $L_{5}=L_{1}, L_{2}$ $=L_{6}=L_{1} / 2, r_{5}=3 r_{u}$; (c) $L_{4}=1.5 L_{1}, L_{8}=L_{9}=L_{1} / 2, r_{5}=r_{8}=3 r_{u}$. 


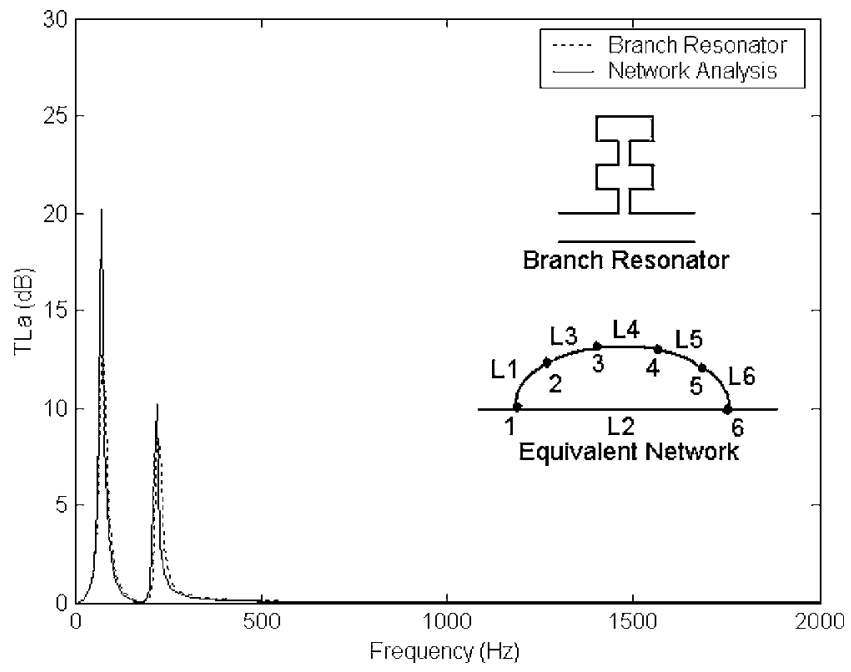

FIG. 9. Transmission loss spectrum for the branch resonator. Comparison with the predicted results from the equivalent network. $L_{1}=0.02 \mathrm{~m}, L_{3}$ $=0.08 \mathrm{~m}, L_{4}=0.02 \mathrm{~m}, L_{5}=0.13 \mathrm{~m}, r_{1}=0.005 \mathrm{~m}, r_{2}=0.02 \mathrm{~m}, r_{3}=0.04 \mathrm{~m}, r_{4}$ $=0.005 \mathrm{~m}, r_{5}=0.04 \mathrm{~m}$. $L_{6}$ is arbitrarily large. $r_{6}=$ arbitrarily small.

In a similar way, the configuration (c) consists of an additional expansion chamber on its lower branch between nodes 6 and 7 (denoted by $L_{8}$ ),

where $L_{4}=1.5 L_{1}, L_{8}=L_{9}=L_{1} / 2$, and $r_{5}=r_{8}=3 r_{u}$.

From the TL curves of Fig. 8 it can be inferred that by inclusion of multiple expansion chambers in the side branches it is possible to enhance the attenuation bandwidth.

The present approach can be used for analysis of other acoustical elements. Figure 9 shows such an example where a branch resonator has been analyzed as a special case of the equivalent network shown in the inset. The corresponding line diagram also has been included in the inset figure. The dimensions (in meters) used are $L_{1}=0.02, L_{3}=0.08, L_{4}$ $=0.02, L_{5}=0.13, r_{1}=0.005, r_{2}=0.02, r_{3}=0.04, r_{4}=0.005, r_{5}$ $=0.04$. In order to simulate the above effect, the radius of the duct between nodes 5 and $6\left(r_{6}\right)$ has been taken as almost zero as has been discussed previously. Length $L_{2}$ was chosen to be $0.02 \mathrm{~m}$ which, in fact, may be assigned any value. It can be seen from the figure that the result from onedimensional analysis correlates well with the prediction from the present approach. The above equivalence motivates analysis of other desired special acoustic elements using an appropriate equivalent network having side branches at many places of the network. By properly choosing the lengths and radii of various ducts and branches, the attenuation behavior can be controlled to obtain better performance over a desired frequency range.

\section{A. Analysis of multiply connected mufflers}

The present approach can be applied to analyze mufflers containing multiply connected components. One simple example of such a muffler and its line diagram representation are shown in Fig. 10. It can be seen from the diagram that the pressure field inside the perforated inlet and in the outer annular duct at plane " $A$ " are interacting with the pressure field in the main duct at plane " $B$." So, this multiple connection

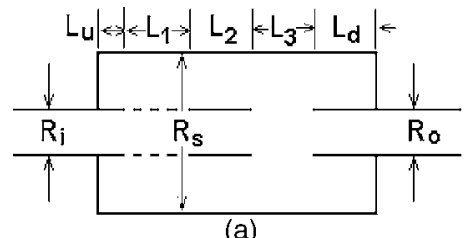

(a)

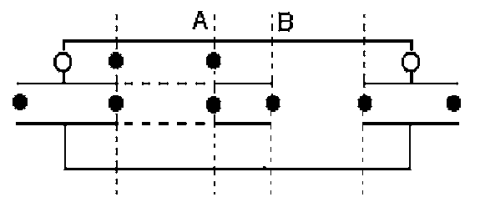

(b)

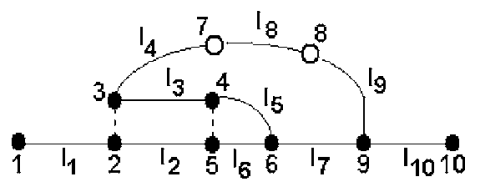

(c)

FIG. 10. (a) Schematic of a multiply connected muffler. (b) Node marking scheme. (c) The branches have been numbered through the algorithm. $L_{u}$ $=L_{d}=0.1 \mathrm{~m}, L_{1}=0.12 \mathrm{~m}, L_{2}=0.12 \mathrm{~m}, L_{3}=0.08 \mathrm{~m}, R_{i}=R_{o}=0.02 \mathrm{~m}, R_{s}$ $=0.05 \mathrm{~m}$.

inside the muffler has been dealt with in the line diagram. Representation of the rigid terminations as long, narrow duct also has been illustrated in this example. The branch representing this long duct is shown by the length " $l_{8}$ " in the figure. The dimensions (in meters) of the muffler are as follows:

$$
\begin{aligned}
& L_{u}=L_{d}=0.1, \quad L_{1}=0.12, \quad L_{2}=0.12, \quad L_{3}=0.08, \\
& R_{i}=R_{o}=0.02, \quad R_{s}=0.05 .
\end{aligned}
$$

The nondimensional perforate impedance, $\zeta$, for the perforated portion of the extended inlet has been calculated according to the following expression: ${ }^{9}$

$$
\zeta=\left[6 \times 10^{-3}+j k\left(t+0.75 d_{h}\right)\right] / \sigma,
$$

where $t$ is the thickness of the duct wall and $d_{h}$ is the diameter of the holes in the perforated region, and $\sigma$ represents the porosity of the perforated portion given by the ratio of open area to the total area of that part. The perforate impedance has been nondimensionalized with respect to the characteristic impedance of air medium. It is to be noted that for these dimensions, the cut-on frequency for the first higher order mode is calculated to be about $2.0 \mathrm{kHz}$. The sound speed is assumed to be $340 \mathrm{~m} / \mathrm{s}$ for the above calculation of cut-on frequency.

The predicted transmission loss spectrum from the present approach has been shown in Fig. 11. A comparison has been made with the results from the 3D-FEM analysis of the same muffler performed with SYSNOISE®, a commercial FEM package. ${ }^{17}$ The results can be seen to be in good agreement for the whole frequency range of interest. The computational time ( 200 millisecond per frequency) for the FEM analysis is not much as compared to the network analysis, for the mesh required for reasonable accurate results for the case under discussion. But, the modeling and the 


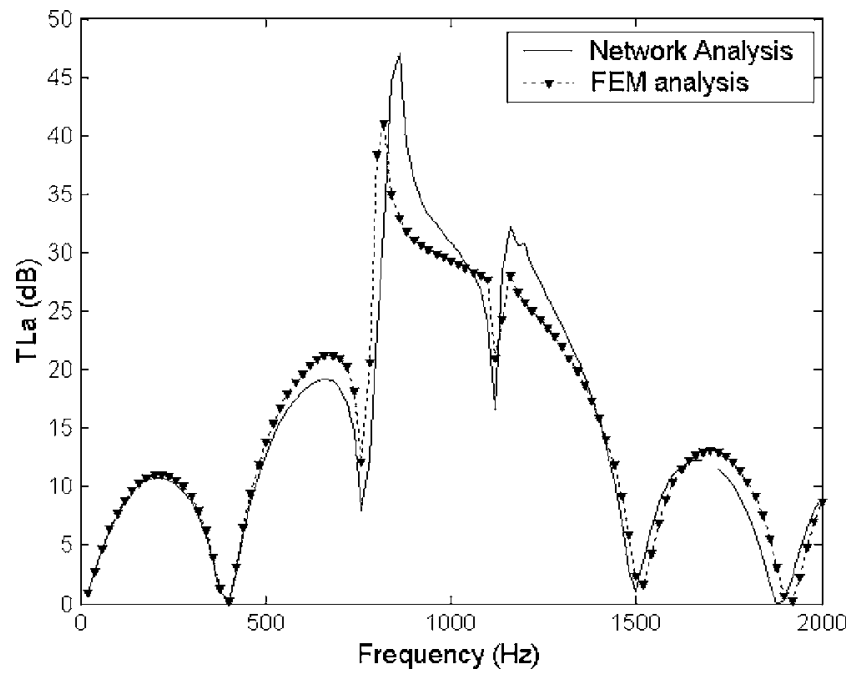

FIG. 11. The predicted TL spectra for the multiply connected muffler shown in Fig. 10. Comparison between 3D FEM and the 1D Network analyses.

mesh generation takes a good amount of time as compared to the data input time for the present analysis. The present analysis even presents a simpler data input scheme as compared to the path fraction algorithm ${ }^{15}$ discussed in the introductory section. So, when it comes to parametric study for optimizing the muffler dimensions, the FEM analysis is not a convenient tool as compared to the network analysis. Figure 12 shows a still more complex multiply connected muffler and the predicted TL spectrum is shown in Fig. 13. It can be seen that the predicted result correlates well with the FEM results. The same perforate impedance expression has been used for both the perforated regions of the muffler. The convenience of using this approach vis-a-vis any FEM package
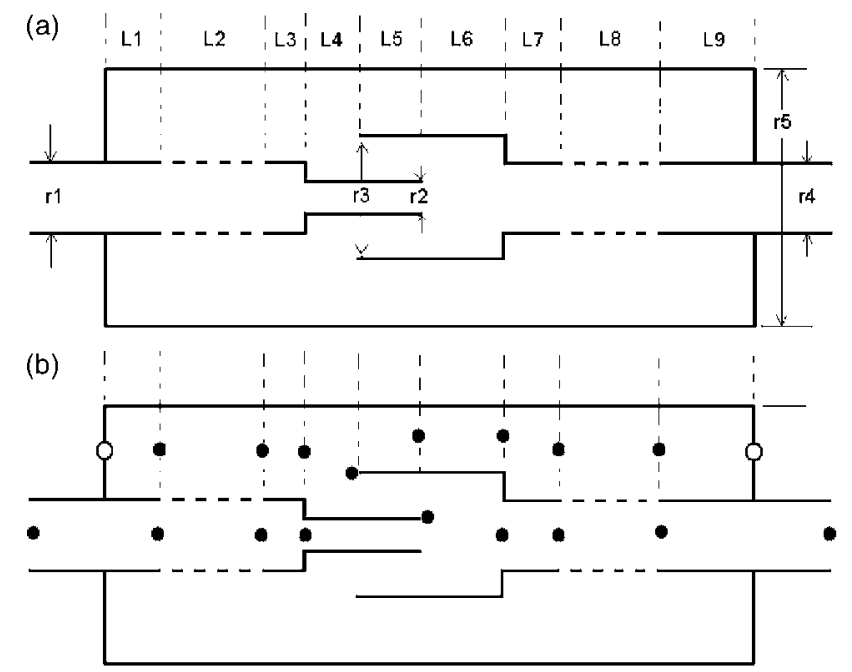

(c)

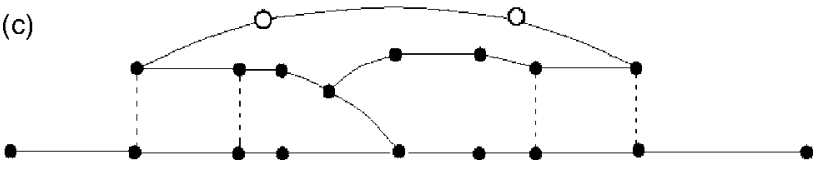

FIG. 12. (a) Schematic of a complex, multiply connected muffler. (b) Node marking scheme. (c) The branches have been numbered through the algorithm. $L_{1}=0.05 \mathrm{~m}, L_{2}=0.131 \mathrm{~m}, L_{3}=0.054 \mathrm{~m}, L_{4}=0.05 \mathrm{~m}, L_{5}=0.05 \mathrm{~m}, L_{6}$ $=0.07 \mathrm{~m}, L_{7}=0.025 \mathrm{~m}, L_{8}=0.091 \mathrm{~m}, L_{9}=0.054 \mathrm{~m}, r_{1}=0.02 \mathrm{~m}, r_{2}=0.01 \mathrm{~m}$, $r_{3}=0.04 \mathrm{~m}, r_{4}=0.02 \mathrm{~m}, r_{5}=0.06 \mathrm{~m}$.

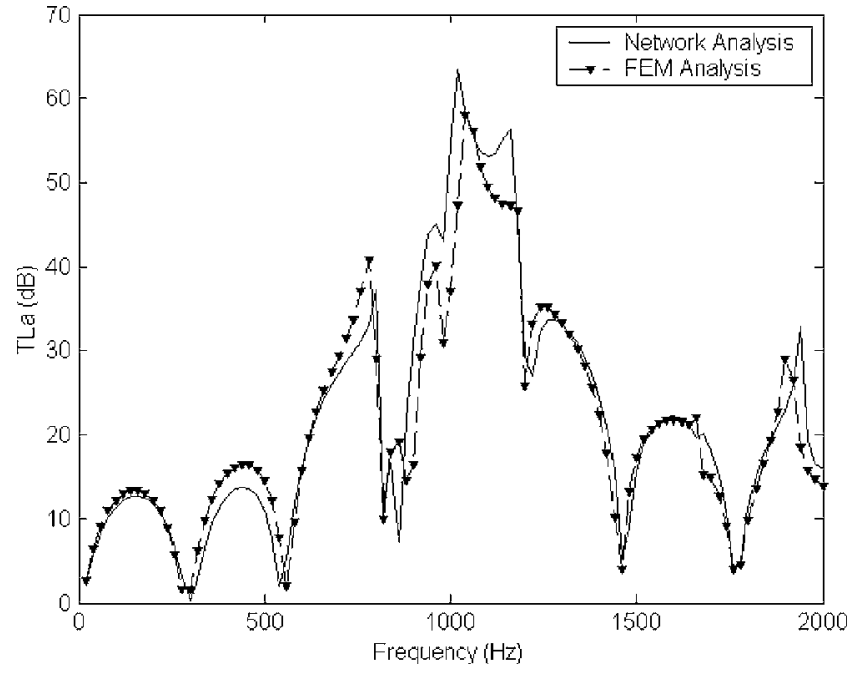

FIG. 13. The predicted TL spectra for the multiply connected muffler shown in Fig. 12. Comparison between 3D FEM and the 1D Network analyses.

can be realized during the parametric study (with respect to geometrical variables) of any system. Though the execution time for the FEM analysis (with a fine mesh) is comparable with that for the present approach, the geometrical model and the FE mesh must be generated afresh for each variation, which is very time consuming, particularly for conducting a parametric study required for design purposes.

\section{B. Example of acoustically equivalent but physically different networks}

Figure 14 shows TL curves for the two configurations shown in the inset. The curves are indeed overlapping. Though the two configurations seem to be physically very different from one another, yet their acoustic behavior, at least in the plane wave range, is identical. The figures of the network in the inset have not been drawn to scale for a quick appreciation of the fact that is being emphasized. For analysis, however, the corresponding lengths between each pair of

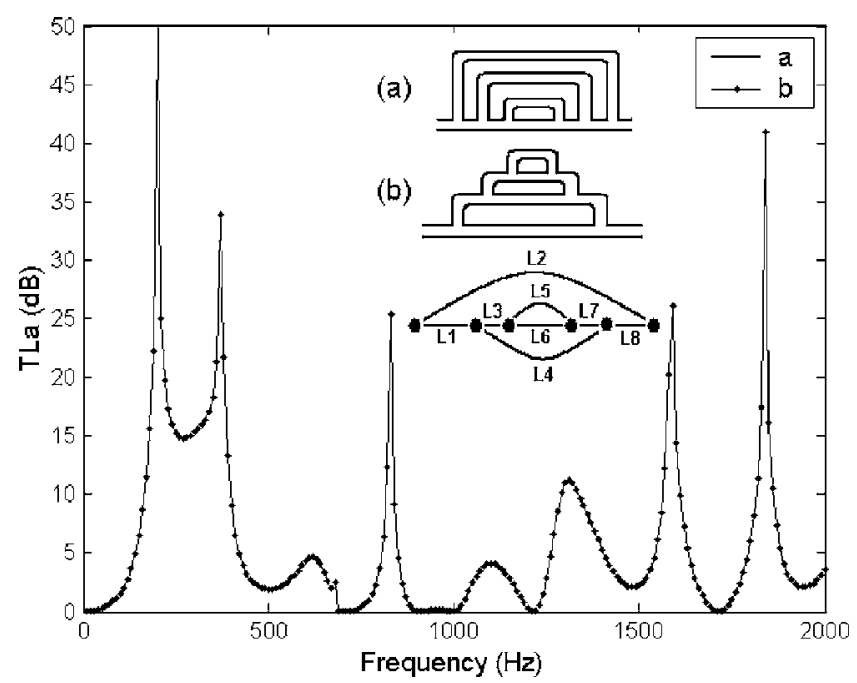

FIG. 14. The predicted TL spectra for two apparently different but acoustically equivalent filter configurations. $L_{1}=0.1 \mathrm{~m}, L_{2}=9 L_{1}, L_{3}=1.5 L_{1}, L_{4}$ $=7 L_{1}, L_{5}=2 L_{1}, L_{6}=3 L_{1}, L_{7}=1.5 L_{1}, L_{8}=L_{1}, r_{1}=0.02 \mathrm{~m}, r_{2}=r_{3}=r_{4}=r_{5}=r_{6}$ $=r_{7}=r_{8}=r_{1}$. 
node have been kept the same for the two networks to become equivalent. Thus the approach outlined here can be used to verify different networks with different physical appearance for their acoustical equivalence.

\section{CONCLUSIONS}

A generalized approach for analyzing the sound attenuation behavior of complex networks (comprising $m$ nodes and $n$ branches) of acoustic filter elements containing gaseous, nondispersive fluids has been presented based on the plane wave assumption. Results for known acoustical elements have been reproduced using the approach as limiting cases, and have been shown to correlate well. The transmission loss predictions have been validated against experimental results from available literature. Higher order modes may affect the accuracy of the approach at higher frequencies for complex networks. The locations of the resonant frequencies however can be predicted with better accuracy. Use of the proposed method has been made to analyze some complex, multiply connected automobile mufflers. Validation has been done through comparison with some analytical closed form results, experimental results, and FEM solutions in some cases where they were available. The approach has also been used to show acoustical equivalence of apparently different networks which in turn can be utilized for designing networks with better space utilization. Apart from networks of simple tubes, networks with multiple expansion chambers at various branches and side branch resonators have been investigated for their pattern of sound attenuation. The results obtained reveal the acoustic resonant frequencies of the configuration under consideration and can be used for analyzing complex network systems with different acoustical elements in the constituent branches.

\section{ACKNOWLEDGMENTS}

Financial support of the Department of Science and Technology of the Government of India for the Facility for Research in Technical Acoustics (FRITA) is gratefully acknowledged.

${ }^{1}$ J. F. W. Herschel, "On the absorption of sound by coloured media viewed in connection with undulatory theory," Philos. Mag. 3, 401-412 (1833).

${ }^{2}$ G. Quincke, "Ueber interferenzapparate für schallwallen," Ann. Phys. Chem. 128, 177-192 (1866).

${ }^{3}$ G. W. Stewart, "The theory of Herschel-Quincke tube," Phys. Rev. 31, 696-698 (1928).

${ }^{4}$ G. W. Stewart and R. B. Lindsay, Acoustics (Van Nostrand, Princeton, NJ, 1930).

${ }^{5}$ G. W. Stewart, "The theory of Herschel-Quincke tube," J. Acoust. Soc. Am. 17, 107-108 (1945).

${ }^{6}$ A. Selamet, N. S. Dickey, and J. M. Novak, "The Herschel-Quincke tube: A theoretical computational and experimental investigation," J. Acoust. Soc. Am. 96, 3177-3185 (1994).

${ }^{7}$ D. D. Davis, Jr., G. M. Stokes, D. Moore, and G. L. Stevens, Jr., "Theoretical and experimental investigation of mufflers with comments on engine-exhaust muffler design," National Advisory Committee for Aeronautics Report No. 1192, 1954.

${ }^{8}$ A. Selamet and V. Easwaran, "Modified Herschel-Quincke tube: Attenuation and resonance for $n$-duct configuration," J. Acoust. Soc. Am. 102, 164-169 (1997).

${ }^{9}$ M. L. Munjal, Acoustics of Ducts and Muffler (Wiley-Interscience, New York, 1987).

${ }^{10}$ M. L. Munjal, "Muffler acoustics," in Formulas of Acoustics, edited by F. P. Mechel (Springer-Verlag, Berlin, 2002), Chap. K.

${ }^{11}$ M. W. Kellogg Company, Design of Piping Systems (Wiley, New York, 1956).

${ }^{12} \mathrm{C}$. W. S. To, "The acoustic simulation and analysis of complicated reciprocating compressor piping systems, I: Analysis technique and parameter matrices of acoustic elements," J. Sound Vib. 96, 175-194 (1984).

${ }^{13} \mathrm{C}$. W. S. To, "The acoustic simulation and analysis of complicated reciprocating compressor piping systems, II: Program structure and applications," J. Sound Vib. 96, 195-205 (1984).

${ }^{14}$ A. Craggs and D. C. Stredulinsky, "Analysis of acoustic wave transmission in a piping network," J. Acoust. Soc. Am. 88, 542-547 (1990).

${ }^{15}$ J. F. Dowling and K. S. Peat, "An algorithm for the efficient acoustic analysis of silencers of any general geometry," Appl. Acoust. 65, 211-227 (2004).

${ }^{16}$ T. Kar and M. L. Munjal, "Generalized analysis of a muffler with any number of interacting ducts," J. Sound Vib. 285, 585-596 (2005).

${ }^{17}$ SYSNOISE Rev 5.6, Users manual, LMS International, 2003. 